\title{
Lacticaseibacillus rhamnosus GG and Saccharomyces cerevisiae boulardii supplementation exert protective effects on human gut microbiome following antibiotic administration in vitro
}

\author{
C. Duysburgh ${ }^{1}$, P. Van den Abbeele ${ }^{1}$, M. Morera ${ }^{2}$ and M. Marzorati ${ }^{1,3^{*}}$ \\ ${ }^{1}$ ProDigest bv, Technologiepark 82, 9052 Ghent, Belgium; ${ }^{2}$ IPSEN Consumer HealthCare SAS, 65 Quai Georges Gorse, \\ 92650 Boulogne Billancourt Cedex, France; ${ }^{3}$ Center of Microbial Ecology and Technology (CMET), Ghent University, \\ Coupure Links 653, 9000 Ghent, Belgium; massimo.marzorati@prodigest.eu
}

Received: 3 September 2020 / Accepted: 7 April 2021

(c) 2021 Wageningen Academic Publishers

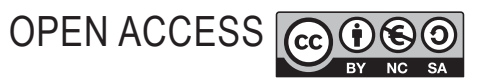

RESEARCH ARTICLE

\begin{abstract}
Antibiotic-induced dysbiosis of the microbial community has been associated with several gastrointestinal symptoms. The impact of repeated administration of Lacticaseibacillus rhamnosus GG (CNCM-I-4798) (formerly known as Lactobacillus rhamnosus GG), Saccharomyces cerevisiae boulardii (CNCM-I-1079) and their combination (associated in Smebiocta/Smectaflora Protect ${ }^{\circ}$ ) in supporting recovery of gut microbiota functionality and composition during and following amoxicillin:clavulanic acid administration was evaluated in vitro. Antibiotic dosage negatively affected SCFA production, coinciding with detrimental effects on Bacteroidetes, Firmicutes and Bifidobacterium spp. in the simulated proximal colon, while Akkermansia muciniphila was significantly reduced in the distal colon. L. rhamnosus GG and S. boulardii were able to thrive in both colon regions upon dosing, with S. boulardii even showing protective effects on the survival of L. rhamnosus GG during antibiotic administration. The impact of the probiotic strains on microbiome recovery revealed that supplementation with L. rhamnosus GG and/or S. boulardii resulted in a stimulating effect on the most abundant bacterial groups within the bacterial community of each donor. For one of the donors tested, co-dosing of L. rhamnosus GG and S. boulardii resulted in superior short-chain fatty acid recovery accompanied by a stronger increase in abundance of Bifidobacteriaceae. Overall, the current study provides first evidence that combined supplementation of L. rhamnosus GG and S. boulardii might be an interesting candidate in limiting detrimental effects of amoxicillin:clavulanic acid on the human gut microbiome, though further studies are warranted to confirm these findings.
\end{abstract}

Keywords: Bifidobacterium, yeast, antibiotic-associated diarrhoea, amoxicillin, clavulanic acid

\section{Introduction}

Although there is growing concern on aspects related to their use, antibiotics are still widely consumed. In 2017, the mean consumption of antibiotics for systemic use in Europe was 21.8 defined daily doses per 1000 inhabitants per day, with penicillins being the most commonly used class of antibiotics with a total consumption rate ranging from 36 to 71\% (European Center for Disease Prevention and Control, 2018). Amoxicillin is the most widespread used penicillin-class antibiotic and is generally combined with clavulanic acid that functions as $\beta$-lactamase inhibitor, thereby avoiding amoxicillin inactivation by $\beta$-lactamasesecreting bacteria (White et al., 2004). While penicillinclass antibiotics are effective in eradicating many pathogens causing bacterial infections, they also adversely affect the indigenous gut microbial community (BrötzOesterhelt and Brunner, 2008). The resulting antibioticinduced colonic imbalance has been linked with several gastrointestinal symptoms. A common disturbance includes antibiotic-associated diarrhea (AAD), which is most often caused by amoxicillin therapy (McFarland, 2008). There is a need to develop strategies that limit antibioticinduced microbial imbalance, thereby potentially reducing 
the incidence of antibiotic-associated gastrointestinal symptoms.

Probiotics are characterised as live microorganisms that confer a health benefit on the host when administered in adequate amounts, probably by supporting maintenance of a balanced gut microbiome (Hill et al., 2014). While probiotic intake has been linked with a variety of physiological effects, several studies reported a potential role of probiotics as preventive and/or therapeutic agent against AAD (Agamennone et al., 2018). Lacticaseibacillus rhamnosus GG (formerly known as Lactobacillus rhamnosus GG) is one of the most well-documented probiotic strains for human application and several studies reported significantly reduced prevalence of AAD upon supplementation of this probiotic strain in vivo (Mantegazza et al., 2018; Szajewska and Kolodziej, 2015; Vanderhoof et al., 1999). In fact, formulations containing a minimum dose of $2 \times 10^{9} \mathrm{cfu}$ L. rhamnosus $\mathrm{GG}$ has been recommended for prevention of AAD in the Netherlands (Agamennone et al., 2018). Furthermore, several other health-promoting properties have been linked with $L$. rhamnosus $\mathrm{GG}$ including regulation of immune function (Capurso, 2019), antipathogenic activity (De Keersmaecker et al., 2006; Spacova et al., 2020) and prevention of atopic disease (Kalliomaki et al., 2001). Another widely used probiotic with proven healthpromoting effects is the yeast Saccharomyces cerevisiae boulardii (Lazo-Vélez et al., 2018). Several human studies reported significant reductions of ADD upon intake S. boulardii in both children and adult patients (Kotowska et al., 2005; McFarland et al., 1995). However, results are conflicting, with Pozzoni et al. (2012) and Ehrhardt et al. (2016) showing absence of effect of $S$. boulardii intake on the development of AAD in elderly hospitalised patients. This strengthens the need to identify specific probiotics that could accompany specific antibiotic therapies.

Despite their clinical relevance, in vivo studies often fail in providing mechanistic insight in how probiotics support host health. In vitro approaches (Minekus et al., 2014, 1999; Molly et al., 1993) offer a suitable alternative as strict control of environmental factors allows evaluation of cross-talk between the indigenous microbial community and the probiotic strain(s) at the site of action. However, as intestinal models depend on human faecal samples to obtain a representative microbiome, it is important to account for inter-individual variation not only during in vivo, but also during in vitro trials (Duysburgh et al., 2019a).

The main objective of the current study was to evaluate the impact of repeated doses of L. rhamnosus GG (CNCM-I-4798), S. boulardii (CNCM-I-1079) and a combination of both strains in supporting recovery of gut microbiota functionality and composition upon cosupplementation with the antibiotic amoxicillin:clavulanic acid. For this purpose, the validated Simulator of the Human
Intestinal Microbial Ecosystem (SHIME ${ }^{\odot}$ ) was used (Van de Wiele et al., 2015; Van den Abbeele et al., 2018), thereby considering the potential inter-individual variability in microbiome composition among human individuals.

\section{Materials and methods}

\section{Chemicals and test product}

All chemicals were obtained from Sigma-Aldrich (Overijse, Belgium) unless stated otherwise. Lallemand Health Solutions Inc. (Blagnac, France) provided the test strains S. boulardii (CNCM-I-1079) and L. rhamnosus GG (CNCM-I-4798), associated in Smebiocta/Smectaflora Protect ${ }^{\circ}$. The products were administered once a day at a daily dose of $1 \times 10^{10}$ L. rhamnosus GG (CNCM-I-4798) and/or $4 \times 10^{9}$ S.boulardii (CNCM-I-1079). The applied dosage corresponds to in vivo dosages as aligned with the AAD dose recommendations (Guarner et al., 2017), considering the in vitro survival rate of the strains during upper gastrointestinal transit (results not shown).

\section{Simulator of the Human Intestinal Microbial Ecosystem}

An adaptation of the SHIME model (ProDigest and Ghent University, Ghent, Belgium) (Molly et al., 1993) was used to simulate the human gastrointestinal tract. The reactor configuration was modified to a QuadSHIME setup allowing the study of four different test conditions in parallel (Dupont et al., 2019). Each arm of the QuadSHIME consisted of a succession of three reactors simulating the different regions of the gastrointestinal tract, i.e. the upper gastrointestinal tract (with subsequent simulation of the gastric and small intestinal phase), the proximal colon (PC) and the distal colon (DC), respectively (Supplementary Figure S1). While the upper gastrointestinal tract reactor is of the fill-and-draw principle, the PC and DC reactors are continuously stirred and have a constant volume and $\mathrm{pH}$ control. The PC was operated at $\mathrm{pH}$ 5.6-5.9 with a retention time of $20 \mathrm{~h}$, while environmental parameters in the DC included a pH-range of 6.6-6.9 and a retention time of 32 $\mathrm{h}$. In order to simulate the luminal and mucus-associated microbiota, mucin-covered microcosms were included in the PC and DC (Supplementary Figure S1) as described by Van den Abbeele et al. (2012). Inoculum preparation, temperature settings, feeding regime and reactor feed composition were implemented from Possemiers et al. (2004). To evaluate the properties of four different test conditions for two healthy human adult donors (donor A: F, 24 years; donor B: F, 25 years), two parallel QuadSHIME experiments were conducted (i.e. each donor was tested in a separate QuadSHIME setup). Informed consent was retrieved for each of the donors to use the faecal samples for the study. Upon inoculation with the faecal inoculum, a two-week stabilisation period was initiated to allow the microbiome to differentiate in the different colonic reactors. 
Subsequently, baseline parameters were established during a one-week control period, after which antibiotic dosage was initiated. Amoxicillin:clavulanic acid (2:1; Toku-E, Sint-Denijs-Westrem, Belgium) was administered to the PC reactors during each feeding cycle, reaching a concentration of $150 \mathrm{mg} /$ day, for a period of 5 days. This antibiotic dosage was determined in order to reach the maximum in vivo quantity that would reach the large intestine. Indeed, in vivo amoxicillin is generally dosed orally at concentrations of 1,500 mg/day (De Velde et al., 2016). Out of this total daily dose, $90 \%$ is absorbed in the small intestine (Legen et al., 2006), resulting in a colonic dose of $150 \mathrm{mg} /$ day. This colonic dose was during the current experiments dosed to the PC reactors, spread over three feeding cycles. Furthermore, in vivo amoxicillin is supplied in combination with clavulanic acid, with clavulanic acid being absorbed for approximately 73\% (EMEA, 2001). Generally, the administered amoxicillin:clavulanic acid ratio is $4: 1$ or $7: 1$, resulting in a ratio of 1.5:1 up to 2.6:1 in the colon when considering absorption rates of $90 \%: 73 \%$. Therefore, an amoxicillin:clavulanic acid ratio of 2:1 was used in this study.

In the aforementioned 5-day antibiotic period, the four arms of each QuadSHIME were operated as follows: (1) control arm (CTRL), only receiving antibiotic; (2) antibiotic + L. rhamnosus GG (CNCM-I-4798) once per day (LGG), (3) antibiotic + S. boulardii (CNCM-I-1079) once per day (SB) and (4) antibiotic + a combination of L. rhamnosus GG (CNCM-I-4798) and $S$. boulardii (CNCM-I-1079) once per day $(\mathrm{LGG}+\mathrm{SB})$. The probiotic strains were administered once per day (i.e. during one feeding cycle) to the $\mathrm{PC}$ reactor, and this approximately $2 \mathrm{~h}$ after the antibiotic had been dosed during that feeding cycle. Administration to the PC reactor was performed as an enteric dosing strategy was envisioned.

After cessation of antibiotic administration, a one-week treatment period followed in which each experimental arm of QuadSHIME was further supplemented with their respective probiotic test products. Finally, a wash-out period of three weeks was initiated in which each QuadSHIME was operated as during the control period to assess the recovery of the gut microbiota.

\section{Enumeration of probiotic strains}

Starting from the control period, samples were collected three times per week from the luminal environment of all colonic reactors to determine the number of $\mathrm{cfu}$ of the probiotic strains by spread plating. Ten-fold dilution series were prepared from these samples in phosphate buffered saline (PBS) and subsequently transferred to petri dishes containing selective agar media. Selective quantification of L. rhamnosus GG was performed through plating on LAMVAB agar media (Hartemink et al., 1997) and plates were subsequently incubated aerobically at $37^{\circ} \mathrm{C}$ for at least $72 \mathrm{~h}$. Selective quantification of $S$. boulardii was performed through plating on Sabouraud agar media supplemented with chloramphenicol $(50 \mathrm{mg} / \mathrm{l})$ and plates were subsequently incubated aerobically at $30{ }^{\circ} \mathrm{C}$ for at least $36 \mathrm{~h}$.

\section{Functionality of gut microbiota}

Starting from the control period, samples for determination of microbial functionality were collected three times per week from both the PC and DC. Measurement of shortchain fatty acid (SCFA) levels, including acetate, propionate, butyrate and total SCFA concentrations, was performed as reported by De Weirdt et al. (2010). Lactate levels were determined using a commercially available enzymatic assay kit (R-Biopharm, Darmstadt, Germany) according to the manufacturer's instructions.

\section{Community composition of gut microbiota}

Starting from the control period, samples for microbiota profiling were collected once per week from the luminal environment in each colonic vessel. DNA was isolated as described by Boon et al. (2003), with some minor modifications as recently reported by Duysburgh et al. (2019b). Quantitative polymerase chain reaction (qPCR) assays for Akkermansia muciniphila, Bacteroidetes and Firmicutes phyla, Lactobacillus spp. and Bifidobacterium spp. were conducted using a QuantStudio 5 Real-Time PCR system (Applied Biosystems, Foster City, CA USA). Each sample was analysed in technical triplicate and outliers (more than $1 \mathrm{C}_{\mathrm{T}}$ difference) were removed. The qPCR for the Firmicutes and Bacteroidetes phyla were performed as described by Guo et al. (2008), while the qPCR for A. muciniphila was conducted as reported in Collado et al. (2007). The qPCR assay for Lactobacillus spp. was adopted from Furet et al. (2009), while the qPCR for Bifidobacterium spp. was performed as reported in Rinttilä et al. (2004). Subsequently, microbiota community composition was assessed by $16 \mathrm{~S}$-targeted Illumina sequencing analysis using isolated DNA originating from samples collected at the end of the control, treatment and wash-out periods. The genomic DNA extracts were diluted in DNase/RNase/ protease-free water to obtain a concentration of $50 \mathrm{ng} / \mu \mathrm{l}$, of which $30 \mu$ l was sent to LGC genomics GmbH (Germany) for library preparation and sequencing on an Illumina Miseq platform with v3 chemistry using primers $341 \mathrm{~F}$ (5'-CCT ACG GGN GGC WGC AG-3') and 785Rmod (5'-GAC TAC HVG GGT ATC TAA KCC-3'), adopted from Klindworth et al. (2013).

\section{Statistics}

Comparison of normally distributed data of the different experimental periods on markers of microbial functionality and microbial community parameters was performed with 
a Student's t-test for pairwise comparisons. Differences were considered significant if $P<0.05$. For the 16S-targeted Illumina sequencing analysis, read assembly and clean-up was largely derived from the MiSeq procedure (Kozich $e t$ al., 2013; Schloss and Westcott, 2011). In brief, the Mothur software (v. 1.40.5) was used to assemble reads into contigs, perform alignment-based quality filtering (alignment to the Mothur-reconstructed SILVA SEED alignment, v. 123), remove chimeras, assign taxonomy using a naïve Bayesian classifier (Wang et al., 2007) and the SILVA NR software v132, and cluster contigs into operational taxonomic units (OTUs) at $97 \%$ sequence similarity. Sequences classified as Eukaryota, Archaea, chloroplasts and mitochondria and sequences that could not be classified, even not at (super) Kingdom level, were omitted. For each OTU, representative sequences were picked as the most abundant sequence within that OTU.

\section{Results}

\section{Colon-region specific colonisation of microbial community}

The main phyla in the microbial community of both donors under investigation included Firmicutes, Bacteroidetes, Actinobacteria, Proteobacteria and Verrucomicrobia and a minor fraction of Synergistetes and Lentisphaerae, though the latter was only detected in microbial community of donor B (Table 1). Specific colonisation patterns were observed in the different colonic regions. Verrucomicrobia, Lentisphaerae and Synergistetes specifically inhabited the DC, which was also observed for several families within the Bacteroidetes (e.g. Rikenellaceae) and Firmicutes (e.g. Ruminococcaceae) phylum, while Bifidobacteriaceae and Veillonellaceae primarily colonised the PC. Further, the biofilm covering the mucin-covered microcosms contained a highly diverse microbial community. A species-specific colonisation of the lumen versus the mucus layer was observed, with Bacteroidaceae specifically colonising the luminal environment, while higher levels of Lachnospiraceae and Ruminococcaceae were observed in the mucus layer. Further, higher abundance of Synergistetes was detected in the mucosal environment, while Verrucomicrobia were specifically present in the lumen. Finally, strong donordependent differences were observed in terms of microbial community composition. While donor A was mainly characterised by high proportions of Bifidobacteriaceae, Lachnospiraceae and Akkermansiaceae (the latter only in the DC), donor B showed high abundance of Veillonellaceae. Furthermore, several bacterial families could specifically be linked to one of the donors under investigation, such as Muribaculaceae and Prevotellaceae for donor A, and Barnesiellaceae, Eubacteriaceae and Victivallaceae for donor B.

\section{Pervasive effects of antibiotic administration on endogenous microbiota}

Antibiotic dosage in the absence of probiotic supplementation (CTRL) resulted in reduced SCFA levels in the PC of both donors tested (Figure 1). While butyrate levels were also reduced in the DC upon antibiotic administration for both donors tested, propionate production was stimulated towards the end of the antibiotic period in this colonic area. On the other hand, acetate levels were slightly reduced in the DC of donor A, while gradually increasing levels were observed during antibiotic dosage for donor $\mathrm{B}$.

In terms of community composition, as assessed by qPCR (Table 2 and Supplementary Table S1), amoxicillin:clavulanic acid dosage resulted in significantly detrimental effects on Bacteroidetes, Firmicutes and Bifidobacterium spp. in the luminal and mucosal PC of both donors tested. Lactobacillus spp. levels were also strongly affected in the luminal PC of donor B, while significantly enhanced lactobacilli levels were observed in the mucosal environment as well as in the proximal lumen and mucus of donor A. In the DC, significantly reduced levels of $A$. muciniphila were observed for both donors tested. In contrast, Bacteroidetes levels were boosted in the DC during antibiotic administration in luminal and mucosal environment, with similar effects observed for the Firmicutes phylum (except in the lumen of donor B). Overall, upon cessation of antibiotic dosage, microbial activity and community composition recovered towards the end of the 28 days of experimental period.

\section{Growth of Lacticaseibacillus rhamnosus GG and Saccharomyces boulardii}

In order to monitor survivability of the administered test strains selective enumeration was performed in all reactors, thereby focussing on the metabolically active cell fraction of the probiotic strains (Figure 2). The combination of L. rhamnosus GG with S. boulardii resulted in a superior survival of $L$. rhamnosus GG during the antibiotic period in both colon regions and for both donors tested. Upon cessation of probiotic supplementation, L. rhamnosus GG washed out from the system, dropping below detection limit for donor A. In contrast, S. boulardii was not affected by antibiotic dosage and nor by L. rhamnosus GG presence/ absence for both donors tested. Upon cessation of product administration, S. boulardii washed out from the system, dropping below detection limit in both colon regions and for both donors tested. For donor A, a faster wash-out was observed in the presence of L. rhamnosus GG. 
Table 1. Colon-region specific colonisation of microbial community. ${ }^{1}$

\begin{tabular}{|c|c|c|c|c|c|c|c|c|c|}
\hline \multirow[t]{3}{*}{ Phylum } & \multirow[t]{3}{*}{ Family } & \multicolumn{4}{|l|}{ Donor A } & \multicolumn{4}{|l|}{ Donor B } \\
\hline & & \multicolumn{2}{|l|}{ L } & \multicolumn{2}{|l|}{$\mathbf{M}$} & \multicolumn{2}{|l|}{ L } & \multicolumn{2}{|l|}{ M } \\
\hline & & PC & $\mathrm{DC}$ & PC & $\mathrm{DC}$ & PC & $\mathrm{DC}$ & PC & $\mathrm{DC}$ \\
\hline \multirow[t]{4}{*}{ Actinobacteria } & Bifidobacteriaceae & $35.38 \%$ & $19.93 \%$ & $36.39 \%$ & $16.44 \%$ & $2.80 \%$ & $2.15 \%$ & $11.12 \%$ & $3.50 \%$ \\
\hline & Coriobacteriaceae & $0.14 \%$ & $0.38 \%$ & $0.25 \%$ & $1.09 \%$ & $0.06 \%$ & $0.12 \%$ & $0.22 \%$ & $4.10 \%$ \\
\hline & Eggerthellaceae & $0.00 \%$ & $0.02 \%$ & $0.00 \%$ & $0.17 \%$ & $0.00 \%$ & $0.04 \%$ & $0.00 \%$ & $0.31 \%$ \\
\hline & Microbacteriaceae & $0.00 \%$ & $0.00 \%$ & $0.00 \%$ & $0.00 \%$ & $0.00 \%$ & $0.00 \%$ & $0.00 \%$ & $0.01 \%$ \\
\hline \multirow[t]{7}{*}{ Bacteroidetes } & Bacteroidaceae & $17.41 \%$ & $12.66 \%$ & $6.45 \%$ & $12.02 \%$ & $16.81 \%$ & $22.96 \%$ & $8.68 \%$ & $23.34 \%$ \\
\hline & Barnesiellaceae & $0.00 \%$ & $0.00 \%$ & $0.00 \%$ & $0.00 \%$ & $0.00 \%$ & $0.06 \%$ & $0.00 \%$ & $0.11 \%$ \\
\hline & Marinifilaceae & $0.00 \%$ & $0.01 \%$ & $0.00 \%$ & $0.33 \%$ & $0.00 \%$ & $0.07 \%$ & $0.00 \%$ & $0.48 \%$ \\
\hline & Muribaculaceae & $0.00 \%$ & $0.08 \%$ & $0.00 \%$ & $2.25 \%$ & $0.00 \%$ & $0.00 \%$ & $0.00 \%$ & $0.00 \%$ \\
\hline & Prevotellaceae & $0.00 \%$ & $0.11 \%$ & $0.00 \%$ & $0.12 \%$ & $0.00 \%$ & $0.00 \%$ & $0.00 \%$ & $0.00 \%$ \\
\hline & Rikenellaceae & $0.03 \%$ & $0.22 \%$ & $0.04 \%$ & $1.37 \%$ & $0.00 \%$ & $0.71 \%$ & $0.00 \%$ & $1.83 \%$ \\
\hline & Tannerellaceae & $2.04 \%$ & $2.23 \%$ & $2.28 \%$ & $2.09 \%$ & $0.95 \%$ & $1.40 \%$ & $3.38 \%$ & $1.03 \%$ \\
\hline \multirow[t]{11}{*}{ Firmicutes } & Acidaminococcaceae & $2.94 \%$ & $1.26 \%$ & $0.47 \%$ & $0.44 \%$ & $0.00 \%$ & $0.02 \%$ & $0.00 \%$ & $0.01 \%$ \\
\hline & Clostridiaceae_1 & $0.00 \%$ & $0.04 \%$ & $0.01 \%$ & $4.10 \%$ & $0.00 \%$ & $0.00 \%$ & $0.00 \%$ & $4.14 \%$ \\
\hline & Enterococcaceae & $0.00 \%$ & $0.00 \%$ & $0.03 \%$ & $0.01 \%$ & $0.16 \%$ & $0.05 \%$ & $0.06 \%$ & $0.03 \%$ \\
\hline & Erysipelotrichaceae & $0.00 \%$ & $0.06 \%$ & $0.00 \%$ & $0.32 \%$ & $0.00 \%$ & $0.19 \%$ & $0.00 \%$ & $0.46 \%$ \\
\hline & Eubacteriaceae & $0.00 \%$ & $0.00 \%$ & $0.00 \%$ & $0.00 \%$ & $0.00 \%$ & $0.28 \%$ & $0.00 \%$ & $1.13 \%$ \\
\hline & Family_XIII & $0.00 \%$ & $0.11 \%$ & $0.00 \%$ & $0.61 \%$ & $0.00 \%$ & $0.09 \%$ & $0.00 \%$ & $0.15 \%$ \\
\hline & Lachnospiraceae & $35.59 \%$ & $31.70 \%$ & $35.12 \%$ & $42.51 \%$ & $11.47 \%$ & $19.97 \%$ & $24.72 \%$ & $25.98 \%$ \\
\hline & Lactobacillaceae & $0.00 \%$ & $0.00 \%$ & $0.00 \%$ & $0.02 \%$ & $0.00 \%$ & $0.00 \%$ & $0.00 \%$ & $0.01 \%$ \\
\hline & Peptostreptococcaceae & $0.00 \%$ & $0.00 \%$ & $0.00 \%$ & $0.09 \%$ & $0.00 \%$ & $0.00 \%$ & $0.00 \%$ & $0.00 \%$ \\
\hline & Ruminococcaceae & $0.09 \%$ & $6.11 \%$ & $0.80 \%$ & $6.52 \%$ & $0.03 \%$ & $2.51 \%$ & $0.30 \%$ & $6.17 \%$ \\
\hline & Veillonellaceae & $0.71 \%$ & $0.41 \%$ & $12.61 \%$ & $1.23 \%$ & $67.51 \%$ & $46.03 \%$ & $51.10 \%$ & $22.26 \%$ \\
\hline Lentisphaerae & Victivallaceae & $0.00 \%$ & $0.00 \%$ & $0.00 \%$ & $0.00 \%$ & $0.00 \%$ & $0.01 \%$ & $0.00 \%$ & $0.11 \%$ \\
\hline \multirow[t]{6}{*}{ Proteobacteria } & Burkholderiaceae & $1.46 \%$ & $0.76 \%$ & $0.75 \%$ & $1.95 \%$ & $0.19 \%$ & $0.66 \%$ & $0.25 \%$ & $1.49 \%$ \\
\hline & Desulfovibrionaceae & $1.97 \%$ & $1.88 \%$ & $3.08 \%$ & $2.51 \%$ & $0.00 \%$ & $1.65 \%$ & $0.01 \%$ & $1.31 \%$ \\
\hline & Enterobacteriaceae & $0.67 \%$ & $0.24 \%$ & $0.37 \%$ & $1.50 \%$ & $0.03 \%$ & $0.06 \%$ & $0.07 \%$ & $0.10 \%$ \\
\hline & Pseudomonadaceae & $1.00 \%$ & $1.06 \%$ & $0.10 \%$ & $0.12 \%$ & $0.00 \%$ & $0.71 \%$ & $0.01 \%$ & $0.23 \%$ \\
\hline & uncultured & $0.54 \%$ & $0.15 \%$ & $1.21 \%$ & $0.06 \%$ & $0.00 \%$ & $0.00 \%$ & $0.00 \%$ & $0.00 \%$ \\
\hline & Xanthomonadaceae & $0.00 \%$ & $0.22 \%$ & $0.00 \%$ & $0.01 \%$ & $0.00 \%$ & $0.01 \%$ & $0.00 \%$ & $0.00 \%$ \\
\hline Synergistetes & Synergistaceae & $0.00 \%$ & $0.02 \%$ & $0.00 \%$ & $0.16 \%$ & $0.00 \%$ & $0.03 \%$ & $0.00 \%$ & $1.25 \%$ \\
\hline Verrucomicrobia & Akkermansiaceae & $0.00 \%$ & $20.28 \%$ & $0.00 \%$ & $1.42 \%$ & $0.00 \%$ & $0.13 \%$ & $0.00 \%$ & $0.14 \%$ \\
\hline
\end{tabular}

\section{Effects of test products on the microbial metabolic activity}

Recovery of microbial metabolic activity following antibiotic dosage was investigated by normalizing SCFA and lactate levels during the final week of the wash-out period to the respective control periods (Table 3 ). These normalised SCFA profiles resulted in treatment-specific observations.
For donor A, a significantly higher production of acetate, propionate and butyrate was observed compared to the antibiotic control incubation (CTRL) upon co-dosing L. rhamnosus GG and S. boulardii in both colon regions (except for acetate in the PC and butyrate in the DC were no significant effect, but a clear trend was observed). Furthermore, the combination of L. rhamnosus GG and $S$. boulardii resulted in synergetic effects as shown 


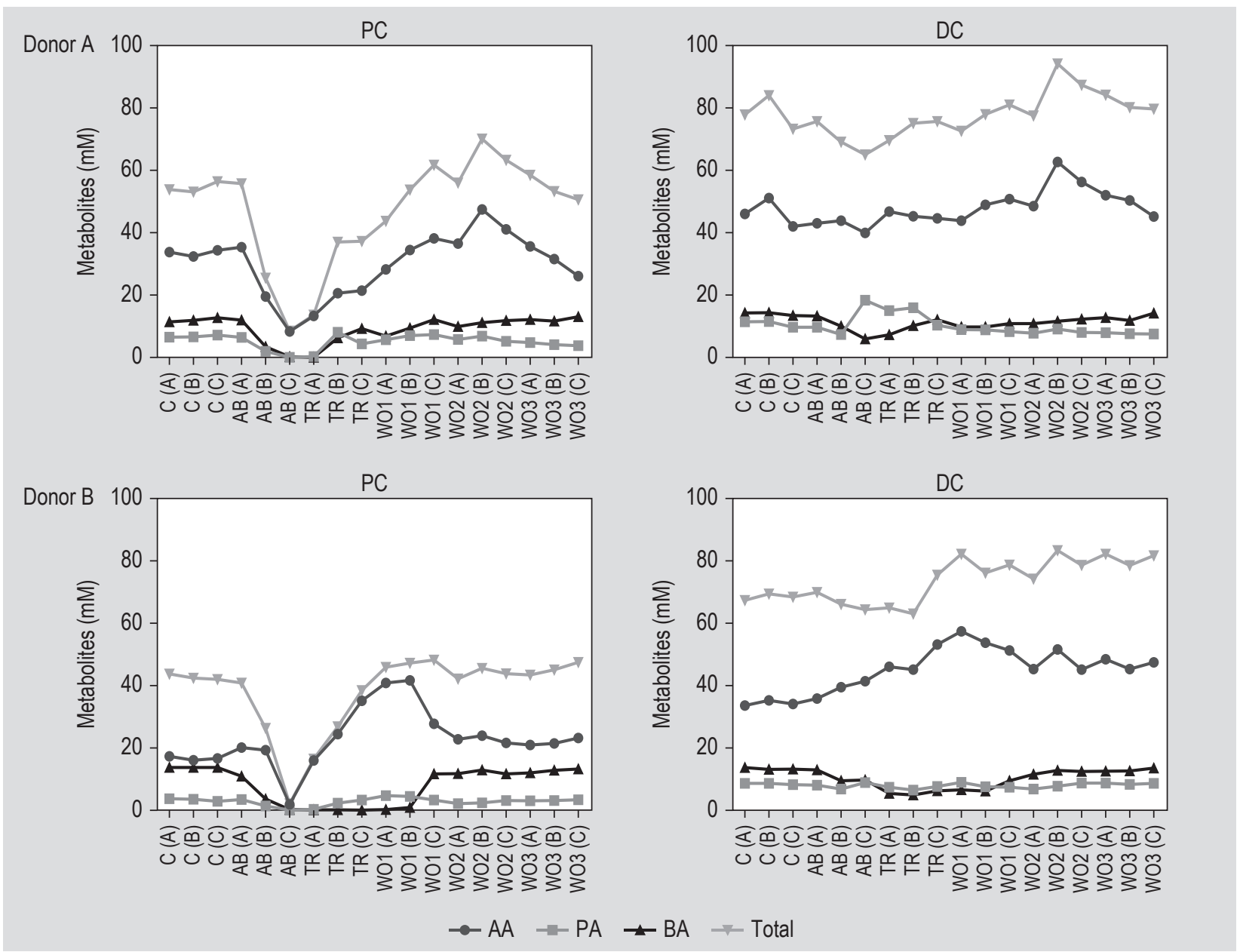

Figure 1. Pervasive effect of antibiotic supplementation on metabolite production. Absolute concentrations (mM) of acetate (AA), propionate (PA), butyrate (BA) and total SCFA in the proximal (PC) and distal colon (DC) reactors for the control unit (CTRL; only receiving antibiotic and thus not supplemented with probiotics during the antibiotic and treatment period) of the SHIME for two different human donors $(n=1)$. Samples were taken one control $(C)$, one antibiotic (AB), one treatment (TR) and three wash-out (WO1, WO2 and WO3) weeks. During each week, three samples (A, B and C) were collected.

by significantly enhanced levels of acetate in the PC, propionate in the $\mathrm{DC}$ and butyrate in both colon regions when comparing the respective individual probiotic supplementation with the combined L. rhamnosus GG and $S$. boulardii condition. For donor B, all test conditions resulted in acetate levels that were higher compared to the control period, however no differences were observed compared to the antibiotic control incubation (CTRL). Similarly, no significant differences were observed between the different test conditions in terms of propionate production. In contrast, administration of S. boulardii resulted in significantly increased butyrate levels compared to the antibiotic control incubation (CTRL) in both colon regions. Finally, dosage of L. rhamnosus GG and its combination with $S$. boulardii significantly reduced lactate production compared to the antibiotic control (CTRL) in the $\mathrm{DC}$ of donor B. For donor A, no significant differences were observed in terms of lactate production.

\section{Effects of test products on microbial community composition}

To assess community composition, a quantitative assessment via qPCR was performed for targeted microbial groups (Figure 3 and Supplementary Figure S2). L. rhamnosus GG supplementation (with or without $S$. boulardii) strongly increased luminal and mucosal Lactobacillus spp. levels in both colon regions for donor A and B, which was probably a direct consequence of the addition of the L. rhamnosus GG strain for these test conditions, thereby indicating that high levels of the probiotic test strain could be maintained in a complex microbial environment. For donor A, levels of Lactobacillus spp. also increased upon amoxicillin:clavulanic acid dosage during the antibiotic control incubation (CTRL), however the observed increase was much smaller compared to the strong increase upon L. rhamnosus GG supplementation. During the wash-out period, Lactobacillus spp. levels decreased (except upon 
Table 2. Pervasive effect of antibiotic administration on bacterial groups as assessed by qPCR. ${ }^{1}$

\begin{tabular}{llll}
$P C$ & & & \\
\hline$C$ & $A B$ & $T R$ & Wo
\end{tabular}

DC

C $\quad A B \quad$ TR

Donor A

Akkermansia muciniphila

Lactobacillus spp.

Bifidobacterium spp.

Bacteroidetes

Firmicutes

Donor $\mathrm{B}$

A. muciniphila

Lactobacillus spp.

Bifidobacterium spp.

Bacteroidetes

Firmicutes

\begin{tabular}{|c|c|c|c|}
\hline below LOD & below LOD & below LOD & below LOD \\
\hline $4.43 \pm 0.29$ & $7.13 \pm 0.04$ & $5.32 \pm 0.09$ & $5.63 \pm 0.64$ \\
\hline $9.73 \pm 0.04$ & $8.44 \pm 0.00$ & $9.77 \pm 0.02$ & $10.00 \pm 0.10$ \\
\hline $9.86 \pm 0.04$ & $6.62 \pm 0.02$ & $9.90 \pm 0.05$ & $9.98 \pm 0.09$ \\
\hline $9.64 \pm 0.01$ & $8.56 \pm 0.01$ & $9.73 \pm 0.04$ & $9.84 \pm 0.14$ \\
\hline below LOD & \pm & & \\
\hline $5.67 \pm 0.00$ & $5.24 \pm 0.02$ & $6.04 \pm 0.02$ & $5.69 \pm 0.14$ \\
\hline $9.10 \pm 0.02$ & $6.61 \pm 0.02$ & $9.86 \pm 0.09$ & $9.52 \pm 0.29$ \\
\hline $9.88 \pm 0.01$ & $6.76 \pm 0.03$ & $10.56 \pm 0.05$ & $9.76 \pm 0.21$ \\
\hline $9.88 \pm 0.01$ & $9.13 \pm 0.01$ & $9.98 \pm 0.02$ & $9.61 \pm 0.16$ \\
\hline
\end{tabular}

\begin{tabular}{|r|r|r|r|}
\hline $9.33 \pm 0.01$ & $5.90 \pm 0.03$ & $8.23 \pm 0.04$ & $8.89 \pm 0.40$ \\
\hline $4.69 \pm 0.13$ & $6.61 \pm 0.04$ & $4.79 \pm 0.08$ & $4.75 \pm 0.35$ \\
\hline $9.88 \pm 0.03$ & $9.21 \pm 0.01$ & $9.85 \pm 0.07$ & $9.62 \pm 0.11$ \\
\hline $10.07 \pm 0.03$ & $10.55 \pm 0.05$ & $10.33 \pm 0.01$ & $9.99 \pm 0.15$ \\
$9.98 \pm 2.49$ & $10.07 \pm 4.06$ & $9.97 \pm 4.31$ & $9.98 \pm 3.29$ \\
\hline & & & \\
\hline $8.02 \pm 0.05$ & below LOD & $\mathbf{6 . 7 2} \pm 0.01$ & $8.14 \pm 3.13$ \\
\hline $5.53 \pm 0.02$ & $4.67 \pm 0.09$ & $\mathbf{5 . 1 0} \pm 0.13$ & $\mathbf{5 . 1 7} \pm 0.09$ \\
\hline $9.29 \pm 0.03$ & $\mathbf{8 . 6 9} \pm 0.01$ & $9.73 \pm 0.03$ & $9.52 \pm 0.16$ \\
\hline $10.26 \pm 0.02$ & $10.65 \pm 0.02$ & $10.47 \pm 0.05$ & $10.22 \pm 0.11$ \\
\hline $10.12 \pm 0.08$ & $9.89 \pm 0.03$ & $10.23 \pm 0.03$ & $10.04 \pm 0.14$ \\
\hline
\end{tabular}

${ }^{1}$ Average Bifidobacterium spp., Lactobacillus spp., A. muciniphila, Bacteroidetes and Firmicutes levels (log 16S rRNA copies/ml) over the control ( $C ; n=1$ in technical triplicate), the antibiotic ( $A B ; n=1$ in technical triplicate), the treatment (TR; $n=1$ in technical triplicate) and the wash-out (WO; $n=3$ in technical triplicate) periods in the luminal proximal (PC) or distal colon (DC) for the control unit (CTRL; only receiving antibiotic) of the SHIME for two different human donors. Data is presented as mean \pm standard deviation. Statistically significant differences relative to the control period are indicated in bold $(P<0.05)$. The intensity of green shading indicates the absolute abundance, normalised for each of the different bacterial groups per colonic region. $\mathrm{LOD}=$ limit of detection.

S. boulardii administration in donor A), with no notable differences being observed between the different test conditions. Further, antibiotic dosage strongly decreased Bifidobacterium spp. levels for both donors tested, especially in the luminal environment. Upon cessation of antibiotic dosage, recovery of Bifidobacterium spp. levels was observed in all treated arms, with no notable differences between the different test conditions.

Microbiota profiling at the main site of fermentation (i.e. luminal PC) (Figure 4), using 16S-targeted Illumina sequencing, confirmed the significantly increased abundance of Lactobacillaceae upon supplementation of $L$. rhamnosus GG (both in the presence and absence of $S$. boulardii) for both donors tested. Similar results, though less apparent, were observed in the mucosal environment (Supplementary Figure S3). As shown by qPCR analyses, this enrichment proved to be a transient effect, as cessation of product administration resulted in a markedly reduced abundance of Lactobacillaceae to levels below the detection limit during the wash-out period. For donor A, antibiotic dosage resulted in reduction of the highly abundant Bifidobacteriaceae family, at the expense of Bacteroidaceae. Furthermore, Proteobacteria were found specifically enriched by antibiotic dosage, as seen by increased abundances of several families belonging to the Proteobacteria phylum for all test conditions. Following product administration, the combination of L. rhamnosus GG and S. boulardii resulted in the strongest increase in Bifidobacteriaceae abundance. In the mucosal environment, the increase in Bifidobacteriaceae was already observed during the treatment period. Moreover, compared to the antibiotic control (CTRL), supplementation of S. boulardii alone or in combination with $L$. rhamnsosus GG resulted in increased levels of Lachnospiraceae, mainly at the expense of Veillonellaceae. Whereas the microbial community of donor A was strongly enriched in Bifidobacteriaceae, the gut microbiota of donor B was characterised by high abundance of Veillonellaceae. Antibiotic dosage drastically reduced Veillonellaceae levels, as seen by the strong decrease in the antibiotic control incubation (CTRL) during the treatment period in both the luminal and mucosal environment. Remarkably, administration of the different test products alleviated this effect, as Veillonellaceae levels remained highly abundant under these test conditions following antibiotic dosage. Following treatment, abundance of several families within the Proteobacteria phylum increased for all test conditions, with a specific increase in Enterobacteriaceae upon S. boulardii supplementation in donor A. Furthermore, some product-specific effects were observed, including increased levels of Lachnospiraceae upon supplementation of $L$. rhamnosus GG and co-dosing L. rhamnosus GG with $S$. boulardii and enhanced Bacteroidaceae levels upon administration of $L$. rhamnosus GG. 
Donor A
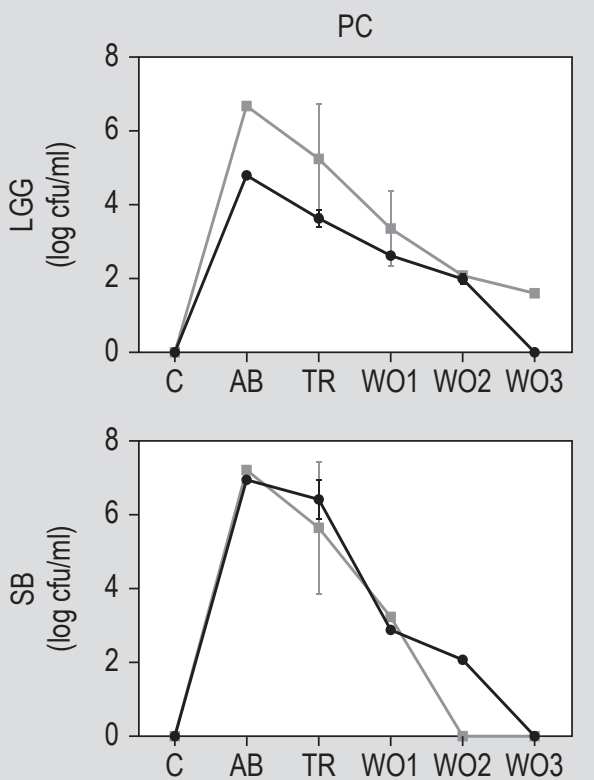

Donor B
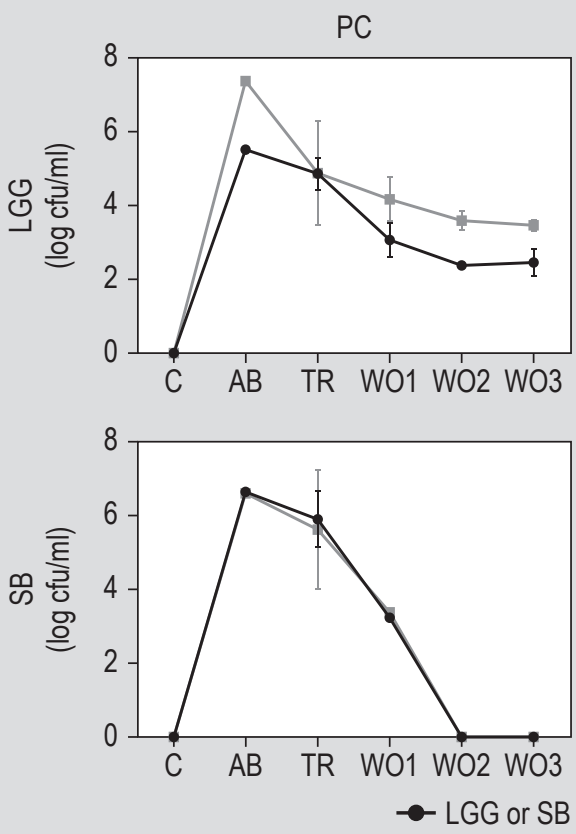
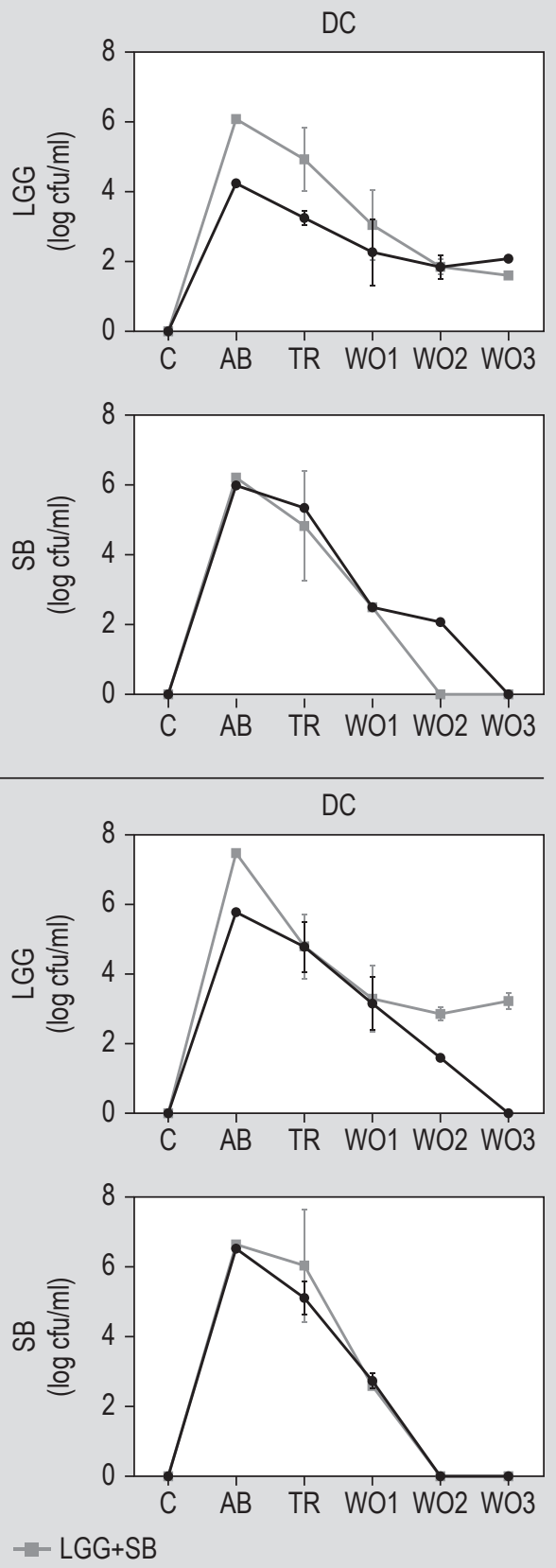

Figure 2. Growth of Lacticaseibacillus rhamnosus GG (LGG) and Saccharomyces boulardii (SB). Average LGG and SB levels (log $\mathrm{cfu} / \mathrm{ml}$ ) over the control (C), the antibiotic (AB), the treatment (TR) and three wash-out (W01, WO2 and WO3) weeks in the luminal proximal (PC) or distal colon (DC) upon administration of the individual LGG or SB strain and their combination (LGG+SB) in the SHIME for two different human donors. Data is presented as mean \pm standard deviation $(n=3)$.

\section{Discussion}

During the current study, the impact of repeated doses of L. rhamnosus GG, S. boulardii and a combination of both strains on recovery of gut microbiota functionality and composition upon co-administration with the antibiotic amoxicillin:clavulanic acid was investigated in vitro. For this purpose, the validated M-SHIME model was used, which allows to create a stable microbial community that is representative for the different colonic regions ( $\mathrm{Liu}$ et al., 2018; Possemiers et al., 2004). The present study confirmed that the M-SHIME model resulted in the creation of a colon region-specific luminal and mucosal microbial community, representative for each of the donors under investigation. Overall, the PC was mainly enriched in bacterial families involved in primary substrate degradation, including Bifidobacteriaceae and Veillonellaceae, while the environmental conditions in the DC selected for microbial groups with specific metabolic functionalities, such as mucin degradation by Akkermansiaceae (Van Herreweghen 
Table 3. Recovery of metabolite production. ${ }^{1}$

\begin{tabular}{|c|c|c|c|c|c|c|c|c|}
\hline & \multicolumn{4}{|l|}{ PC } & \multicolumn{4}{|l|}{$\mathrm{DC}$} \\
\hline & CTRL & LGG & SB & LGG+SB & C & LGG & SB & LGG+SB \\
\hline \multicolumn{9}{|l|}{ Donor A } \\
\hline Acetate (mM) & $-2.46 \pm 4.79 a, b$ & $-1.92 \pm 1.94^{a}$ & $1.03 \pm 1.39$ a & $6.58 \pm 1.71^{b}$ & $2.81 \pm 3.58^{a}$ & $6.28 \pm 3.75^{a, b}$ & $8.87 \pm 3.08^{a, b}$ & $11.40 \pm 2.43^{b}$ \\
\hline Propionate (mM) & $-2.56 \pm 0.52^{a}$ & $-2.50 \pm 0.36^{a, b}$ & $-1.39 \pm 0.92^{a}$ & $0.10 \pm 0.83^{b}$ & $-3.18 \pm 0.24^{a}$ & $-3.58 \pm 0.38^{a}$ & $-3.19 \pm 1.00^{a}$ & $0.89 \pm 0.80^{b}$ \\
\hline Butyrate (mM) & $0.25 \pm 0.72^{a}$ & $1.50 \pm 0.61^{b}$ & $2.33 \pm 0.18^{b}$ & $4.75 \pm 0.26$ & $-1.09 \pm 1.20 a, b$ & $-2.49 \pm 0.82^{a}$ & $-0.75 \pm 0.64^{a}$ & $2.97 \pm 0.53^{b}$ \\
\hline Lactate (mM) & $-0.46 \pm 0.10$ & $-0.52 \pm 0.06$ & $-0.50 \pm 0.02$ & $-0.53 \pm 0.02$ & $-0.16 \pm 0.06^{a}$ & $-0.03 \pm 0.07^{b}$ & $0.28 \pm 0.63^{a, b}$ & $1.04 \pm 0.83^{a, b}$ \\
\hline Total SCFA (mM) & $-0.33 \pm 4.06^{a, b}$ & $0.83 \pm 2.00^{a}$ & $3.37 \pm 1.46^{a}$ & $12.42 \pm 2.36^{b}$ & $2.98 \pm 2.49^{a}$ & $6.90 \pm 4.06^{a, b}$ & $11.11 \pm 4.31$ a,b & $16.85 \pm 3.29 b$ \\
\hline \multicolumn{9}{|l|}{ Donor B } \\
\hline Acetate (mM) & $5.21 \pm 1.20$ & $21.54 \pm 10.39$ & $8.85 \pm 1.90$ & $9.89 \pm 3.66$ & $12.84 \pm 1.62$ & $15.59 \pm 4.26$ & $9.44 \pm 5.07$ & $13.19 \pm 3.13$ \\
\hline Propionate (mM) & $-0.21 \pm 0.17$ & $2.80 \pm 1.64$ & $0.51 \pm 0.38$ & $0.57 \pm 0.28$ & $0.07 \pm 0.22$ & $0.89 \pm 0.63$ & $0.45 \pm 1.03$ & $-0.03 \pm 0.41$ \\
\hline Butyrate (mM) & $-1.05 \pm 0.65^{a}$ & $-0.94 \pm 0.56^{a}$ & $0.64 \pm 0.81^{b}$ & $-0.09 \pm 0.17^{a, b}$ & $-0.47 \pm 0.53^{a}$ & $-1.60 \pm 0.49^{a}$ & $0.73 \pm 0.26^{b}$ & $-0.92 \pm 0.31^{a}$ \\
\hline Lactate (mM) & $0.29 \pm 0.36^{a, b}$ & $-0.09 \pm 0.16^{a}$ & $0.19 \pm 0.19^{b}$ & $0.39 \pm 0.27^{b}$ & $-0.02 \pm 0.06^{a}$ & $-0.26 \pm 0.03^{b}$ & $0.01 \pm 0.18^{a, b}$ & $-0.16 \pm 0.09 b$ \\
\hline Total SCFA (mM) & $2.62 \pm 2.03$ & $20.39 \pm 11.85$ & $8.85 \pm 1.51$ & $8.72 \pm 3.49$ & $12.48 \pm 2.02$ & $13.33 \pm 4.91$ & $9.56 \pm 6.07$ & $12.20 \pm 2.94$ \\
\hline \multicolumn{9}{|c|}{$\begin{array}{l}{ }^{1} \text { Average normalised concentration of acetate, propionate, butyrate, lactate and total short chain fatty acid (SCFA, mM) associated to the } \\
\text { administration of Lacticaseibacillus rhamnosus GG (LGG), Saccharomyces boulardii (SB) and their combination (LGG+SB) compared to a control } \\
(\mathrm{CTRL}) \text { in the proximal }(\mathrm{PC}) \text { and distal colon }(\mathrm{DC}) \text { during the final wash-out week (WO3) in the SHIME for two different human donors. Data is } \\
\text { presented as mean } \pm \text { standard deviation }(n=3) \text {. Statistically significant differences between the different conditions in each of the colonic environments } \\
\text { are indicated with different letters }(P<0.05) \text {. The intensity of green shading indicates the absolute abundance, normalised for each of the different } \\
\text { metabolites per colonic region. }\end{array}$} \\
\hline
\end{tabular}

et al., 2017). This colon-region specificity confirms previous findings by Van den Abbeele et al. (2010). Further, the mucosal biofilm has been shown to be specifically colonised by butyrate-producing members of the Firmicutes phylum (Van den Abbeele et al., 2013) as well as species from the Synergistetes phylum (Liu et al., 2018), as demonstrated during the current study. Overall, the establishment of a representative microbiome in the M-SHIME model provided an excellent tool for performing mechanistic research to unravel effects on the human gut microbiome.

Antibiotic administration strongly affected the in vitro microbial community, resulting in diminished levels of SCFA and detrimental effects in terms of community composition. Overall effects were strongest in the PC, probably due to dilution of antibiotic treatment or microbial degradation of amoxicillin:clavulanic acid when moving to the DC. Butyrate production was most strongly affected, showing significant reductions in both colon regions upon amoxicillin:clavulanic acid dosage, confirming findings of in vitro work performed by Ladirat et al. (2014b). Moreover, the abundance of Lachnospiraceae and Ruminococcaceae, two families containing several butyrate-producing species, decreased significantly following amoxicillin treatment in vivo (Ladirat et al., 2014a; Tsitko et al., 2019), which was for the former bacterial family also observed during the current study in the luminal environment of both donors tested. In concordance with previous in vivo studies (Ladirat et al., 2014a; Soldi et al., 2019), Bifidobacterium spp. levels were also strongly affected by the amoxicillin:clavulanic acid administration. Propionate production was stimulated in the DC coinciding with enhanced Bacteroidetes proportions towards the end of the antibiotic period. While several studies have reported a similar stimulation of several members of the Bacteroidetes phylum following amoxicillin administration (Barc et al., 2004; Panda et al., 2014), others showed reduced Bacteroidetes levels as observed in the PC in the current study (Ladirat et al., 2014b; Tsitko et al., 2019). It can be hypothesised that the suppression of Bifidobacterium spp. and butyrateproducing microorganisms by antibiotic dosage created a competitive advantage for other bacterial species, including members of the Bacteroidetes phylum. This might induce enrichment of the Gram-negative bacterial flora (such as several groups within the Bacteroidetes phylum), which has been linked with several side effects such as the induction of diarrhoea (Ramamurthy et al., 2013). Overall, it can be concluded that amoxicillin:clavulanic acid dosage exerted a detrimental effect on the in vitro microbial community, in accordance with in vivo data, which might be related with the occurrence of AAD.

In order to link the potential effect of $L$. rhamnosus GG and $S$. boulardii on the recovery of the gut microbiome during and following antibiotic supplementation, the presence of viable L. rhamnosus GG and S. boulardii 
Donor A
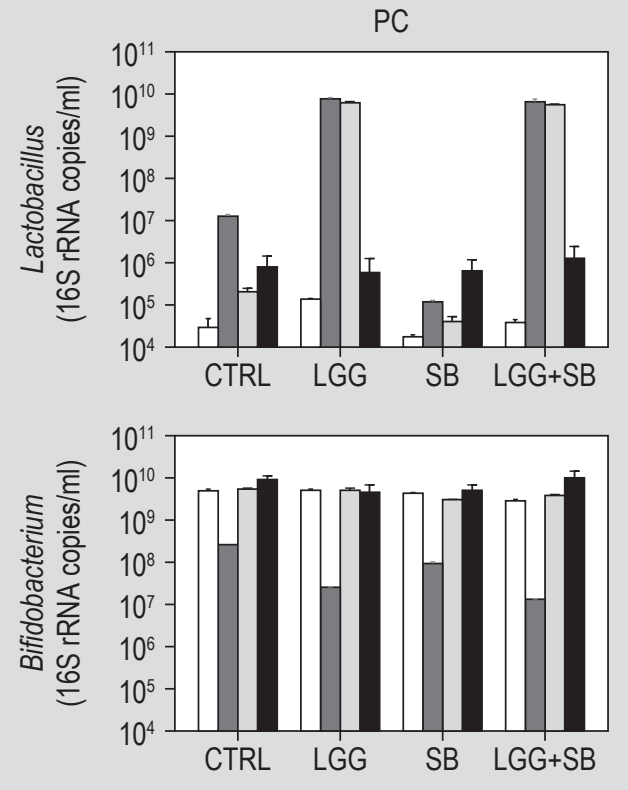

Donor B
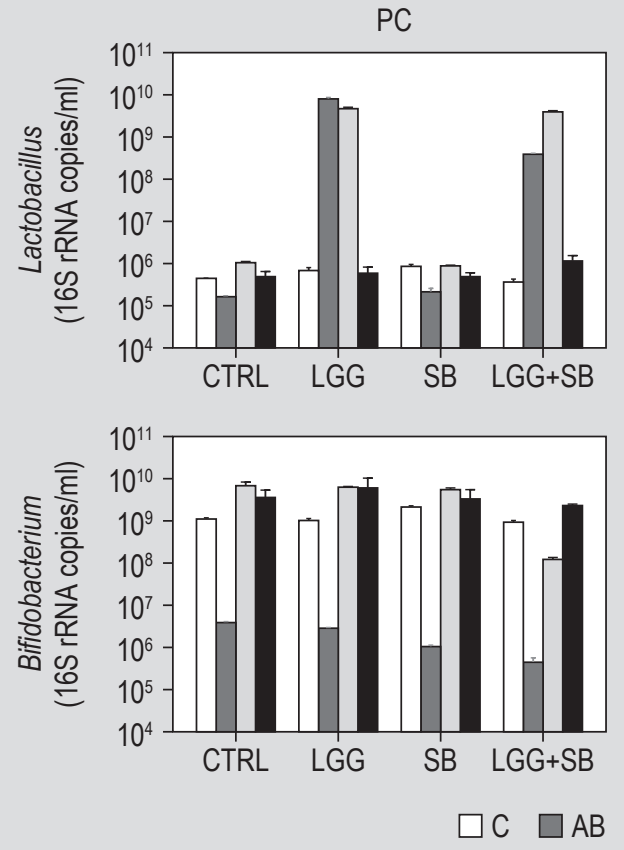
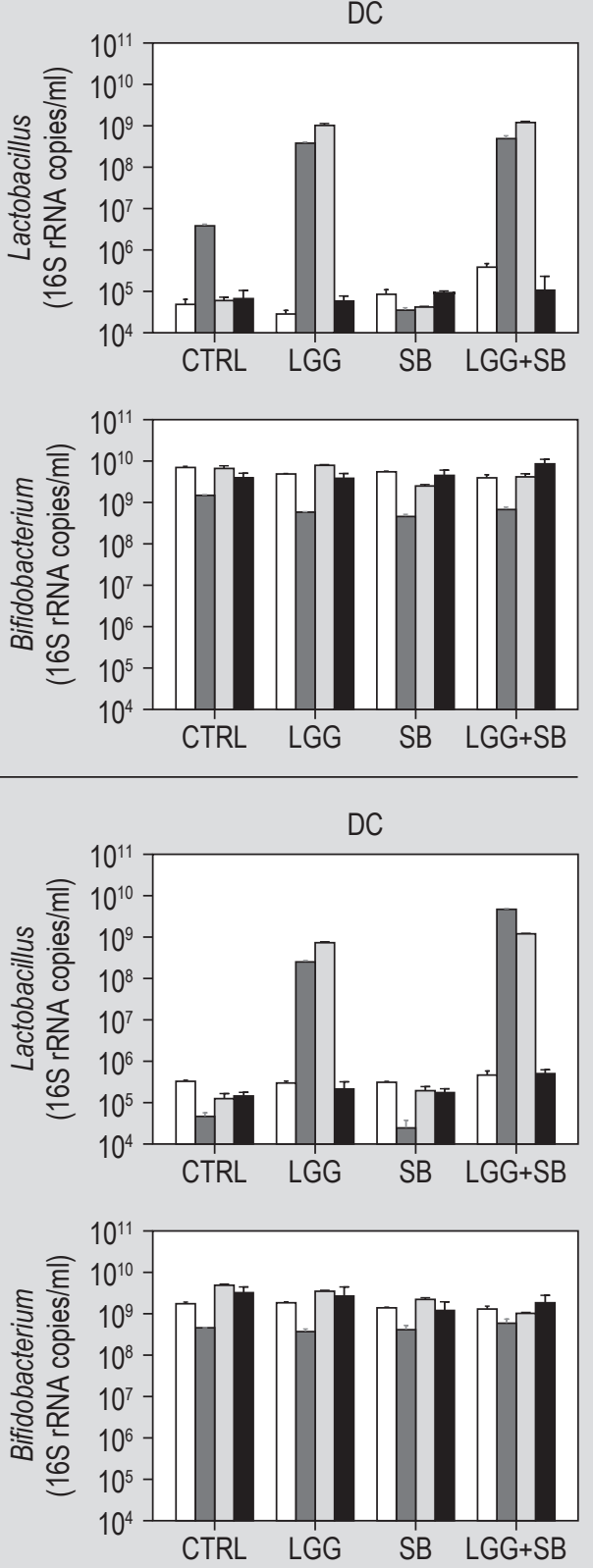

$\square$ TR $\square$ WO

Figure 3. Luminal microbial community composition as assessed via qPCR. Average Bifidobacterium spp. and Lactobacillus spp. levels (log 16S rRNA copies $/ \mathrm{mL}$ ) associated to the administration of Lacticaseibacillus rhamnosus GG (LGG), Saccharomyces boulardii (SB) and their combination (LGG+SB) compared to a control (CTRL) in the luminal proximal (PC) or distal colon (DC) during the control ( $C ; n=1$ in technical triplicate), the antibiotic ( $A B ; n=1$ in technical triplicate), the treatment (TR; $n=1$ in technical triplicate) and the wash-out (WO; $n=3$ in technical triplicate) periods in the SHIME for two different human donors. Data is presented as mean \pm standard deviation.

cells was assessed throughout the experimental period. Selective enumeration pointed out that viable L. rhamnosus GG cells were specifically detected in the SHIME units where L. rhamnosus GG was administered. The survival of L. rhamnosus GG during antibiotic dosage was enhanced upon co-supplementation with $S$. boulardii versus the unit where L. rhamnosus GG was administered alone, and this in both colon regions and for both donors tested. Recently, it was shown by our research group that no antagonistic effects between L. rhamnosus GG and S. boulardii were observed when cultivated together (Moens et al., 2019). The current study further complements these observations, showing protective effects of $S$. boulardii on survival of L. rhamnosus GG during antibiotic dosage. Further, high 


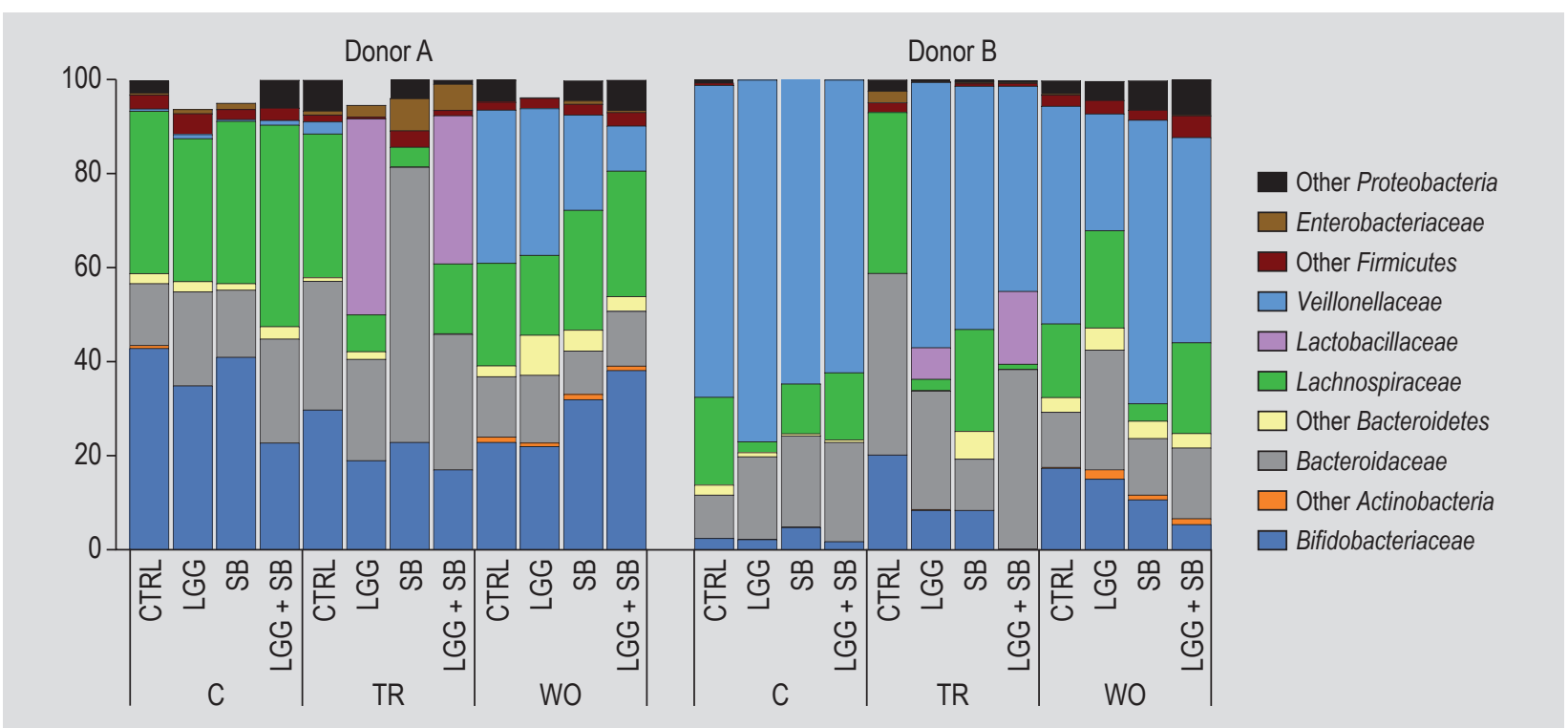

Figure 4. Luminal microbial community composition as assessed via $16 \mathrm{~S}$-targeted Illumina sequencing. Abundance $(\%)$ at microbial family level associated to the administration of Lacticaseibacillus rhamnosus GG (LGG), Saccharomyces boulardii (SB) and their combination (LGG+SB) compared to a control (CTRL) in the luminal proximal colon (PC) at the end of the control period (C), treatment period (TR) and wash-out period (WO) in the SHIME for two different human donors ( $n=1)$.

levels of viable $S$. boulardii cells were detected in the SHIME units where $S$. boulardii was administered and these levels were not affected by the antibiotic dosage, confirming literature findings (Neut et al., 2017), regardless of the presence/absence of L. rhamnosus GG. Upon cessation of the product administration, both test strains washed out from the system. It has been shown that persistence of individual strains varies in a strain-specific manner in vivo (Tremblay et al., 2020). Indeed, while S. boulardii is only to persist in the colonic environment for hours upon cessation of dosing, L. rhamnosus GG can persevere for several weeks in the colonic environment (McFarland and Elmer, 2005). Overall, these findings demonstrated that the strains were not only dosed but also capable to thrive in both colon regions of both donors tested, thus potentially resulting in beneficial effects on antibiotic-induced dysbiosis.

The impact of the probiotic test strains on the recovery of microbial functionality and composition showed donordependent differences. 16S-targeted Illumina sequencing revealed that supplementation of L. rhamnosus GG and/ or $S$. boulardii resulted in a protective effect on the most abundant bacterial groups within the bacterial community of each donor upon antibiotic dosage. Donor A was mainly characterised by high proportions of Bifidobacteriaceae and Lachnospiraceae, with both bacterial groups being highly affected by antibiotic dosage. Upon cessation of antibiotic administration, Lachnospiraceae recovered in all test conditions, with higher levels observed upon probiotic supplementation, especially for addition of S. boulardii and its combination with L. rhamnosus GG. Supplementation of $S$. boulardii has been reported to lead to a faster recovery of the gut microbiome following a state of dysbiosis, including positive effects on Lachnospiraceae levels (Moré and Swidsinski, 2015). Furthermore, co-dosing L. rhamnosus GG and S. boulardii resulted in superior SCFA recovery compared to the antibiotic control, accompanied by a stronger increase in abundance of Bifidobacteriaceae. This confirmed previous results from our research group, which demonstrated that combination of L. rhamnosus GG and $S$. boulardii improved microbiota functionality under impaired conditions (Moens et al., 2019). Donor B instead was characterised by a higher abundance of Veillonellaceae in its healthy state, levels of which were drastically reduced following antibiotic dosage in the control arm, while supplementation of the different probiotic test strains preserved Veillonellaceae levels, indicating a potential protective effect of L. rhamnosus GG and S. boulardii on Veillonellaceae levels during antibiotic dosage for this particular donor. Collectively, these results indicate that effects of antibiotic co-treatment with L. rhamnosus GG and S. boulardii on gut microbiota functionality and composition may differ substantially depending on the baseline composition of the gut microbiota of each subject, although reinforcing the hypothesis that combined intake of L. rhamnosus GG and S. boulardii might stimulate microbiome recovery following antibiotic dosage.

Some potential limitations of the study are acknowledged. While inter-individual variations were evaluated, only two donors were selected for the current study. However, it is important to note that the human microbial community composition is generally characterised by large interindividual differences (Eckburg et al., 2005) and this 
should therefore also be taken into account upon using in vitro gut models. It has been reported that the gut microbial community of healthy individuals naturally recovers following antibiotic administration, reaching a near-baseline composition within 45 days following a short antibiotic intervention period (Palleja et al., 2018). In the current study, a parallel antibiotic control was included to map natural microbiome recovery following a 5-day amoxicillin:clavulanic acid dosage in vitro. Near-full recovery of microbial metabolic activity and community composition was observed during the post-intervention period of 28 days. This fast recovery of the microbial community during the present in vitro study might therefore have obscured potential effects of L. rhamnosus, S. boulardii and their combination on microbiome recovery during the post-intervention period. Furthermore, while the gut microbial community generally recovers to its healthy state in 45 days due to its resilience, long-term alterations may persist in some subjects explaining susceptibility to AAD or other dysbiosis-related diseases (De La Cochetière et al., 2005; Sommer and Bäckhed, 2013). Furthermore, hostmicrobiome interactions were not investigated during the current study. While the current study has shown that not only the luminal microbial community was affected by the administered test products, but that also the mucusassociated microbiota was impacted, these results point to potential interesting interactions with the intestinal epithelial cells. Combining samples obtained from the SHIME model with a co-culture human cell model (Daguet et al., 2016), could have provided insight on the immunemodulatory properties of L. rhamnosus, S. boulardii and their combination following antibiotic dosage.

In conclusion, administration the antibiotic amoxicillin: clavulanic acid exerted a detrimental effect on the human gut microbiome in the simulated gastrointestinal tract. While recovery of the microbial community was observed at the end of the $28 \mathrm{~d}$ experimental period, supplementation of $L$. rhamnosus GG and $S$. boulardii positively impacted colonic balance, though in a donor-dependent fashion. Evidence was provided that co-dosing of L. rhamnosus GG and S. boulardii might additionally stimulate recovery of microbial functionality and composition following amoxicillin:clavulanic acid dosage, which could provide an interesting approach for antibiotic-related gastrointestinal symptoms in the clinical environment such as in the protection against AAD. However, future research is warranted to confirm these findings.

\section{Supplementary material}

Supplementary material can be found online at https:// doi.org/10.3920/BM2020.0180.

Figure S1. Design of SHIME experiment.
Figure S2. Mucosal microbial community composition as assessed via qPCR.

Figure S3. Mucosal microbial community composition as assessed via $16 \mathrm{~S}$-targeted Illumina sequencing.

Table S1. Pervasive effect of antibiotic administration on mucosal bacterial groups as assessed by qPCR.

\section{Acknowledgements}

This work was sponsored by IPSEN Consumer HealthCare SAS. The authors thank the donors who kindly supplied a faecal sample for the purpose of the study. Further, the authors thank Dr Thomas A. Tompkins, Dr Annie Tremblay and Dr Frédéric Moens for critically reviewing the manuscript.

\section{Conflict of interest}

Mireia Morera is an employee of IPSEN Consumer HealthCare SAS.

\section{References}

Agamennone, V., Krul, C.A.M., Rijkers, G. and Kort, R., 2018. A practical guide for probiotics applied to the case of antibioticassociated diarrhea in the Netherlands. BMC Gastroenterology 18: 103.

Barc, M.C., Bourlioux, F., Rigottier-Gois, L., Charrin-Sarnel, C., Janoir, C., Boureau, H., Dore, J. and Collignon, A., 2004. Effect of amoxicillin-clavulanic acid on human fecal flora in a gnotobiotic mouse model assessed with fluorescence hybridization using groupspecific $16 \mathrm{~S}$ rRNA probes in combination with flow cytometry. Antimicrobial Agents and Chemotherapy 48: 1365-1368.

Boon, N., Top, E.M., Verstraete, W. and Siciliano, S.D., 2003. Bioaugmentation as a tool to protect the structure and function of an activated-sludge microbial community against a 3-chloroaniline shock load. Applied and Environmental Microbiology 69: 1511-1520.

Brötz-Oesterhelt, H. and Brunner, N.A., 2008. How many modes of action should an antibiotic have? Current Opinion in Pharmacology 8: 564-573.

Capurso, L., 2019. Thirty years of Lactobacillus rhamnosus GG: a review. Journal of Clinical Gastroenterology 53: S1-S41.

Collado, M.C., Derrien, M., Isolauri, E., de Vos, W.M. and Salminen, S., 2007. Intestinal integrity and Akkermansia muciniphila, a mucindegrading member of the intestinal microbiota present in infants, adults, and the elderly. Applied and Environmental Microbiology 73: 7767-7770.

Daguet, D., Pinheiro, I., Verhelst, A., Possemiers, S. and Marzorati, M., 2016. Arabinogalactan and fructooligosaccharides improve the gut barrier function in distinct areas of the colon in the simulator of the human intestinal microbial ecosystem. Journal of Functional Foods 20: 369-379. 
De Keersmaecker, S.C., Verhoeven, T.L., Desair, J., Marchal, K., Vanderleyden, J. and Nagy, I., 2006. Strong antimicrobial activity of Lactobacillus rhamnosus GG against Salmonella Typhimurium is due to accumulation of lactic acid. FEMS Microbiology Letters 259: 89-96.

De La Cochetière, M.F., Durand, T., Lepage, P., Bourreille, A., Galmiche, J.P. and Doré, J., 2005. Resilience of the dominant human fecal microbiota upon short-course antibiotic challenge. Journal of Clinical Microbiology 43: 5588-5592.

De Velde, F., de Winter, B.C., Koch, B.C., van Gelder, T. and Mouton, J.W., 2016. Non-linear absorption pharmacokinetics of amoxicillin: consequences for dosing regimens and clinical breakpoints. Journal of Antimicrobial Chemotherapy 71: 2909-2917.

De Weirdt, R., Possemiers, S., Vermeulen, G., Moerdijk-Poortvliet, T.C.W., Boschker, H.T.S., Verstraete, W. and Van de Wiele, T., 2010. Human faecal microbiota display variable patterns of glycerol metabolism. FEMS Microbiology Ecology 74: 601-611.

Dupont, D., Alric, M., Blanquet-Diot, S., Bornhorst, G., Cueva, C., Deglaire, A., Denis, S., Ferrua, M., Havenaar, R., Lelieveld, J., Mackie, A.R., Marzorati, M., Menard, O., Minekus, M., Miralles, B., Recio, I. and Van den Abbeele, P., 2019. Can dynamic in vitro digestion systems mimic the physiological reality? Critical Reviews in Food Science and Nutrition 59: 1546-1562.

Duysburgh, C., Van Den Abbeele, P., Bothe, M., Stover, J., KuchinkaKoch, A., Schwejda-Guettes, S. and Marzorati, M., 2019a. Lactulose crystals beneficially affect community composition along entire human colon in vitro, resulting in donor-dependent prebiotic effects at metabolic level. International Journal of Nutrition and Food Sciences 8: 10

Duysburgh, C., Van den Abbeele, P., Krishnan, K., Bayne, T.F. and Marzorati, M., 2019b. A synbiotic concept containing spore-forming Bacillus strains and a prebiotic fiber blend consistently enhanced metabolic activity by modulation of the gut microbiome in vitro. International Journal of Pharmaceutics: X 1: 100021.

Eckburg, P.B., Bik, E.M., Bernstein, C.N., Purdom, E., Dethlefsen, L., Sargent, M., Gill, S.R., Nelson, K.E. and Relman, D.A., 2005. Diversity of the human intestinal microbial flora. Science 308: 1635-1638.

Ehrhardt, S., Guo, N., Hinz, R., Schoppen, S., May, J., Reiser, M., Schroeder, M.P., Schmiedel, S., Keuchel, M., Reisinger, E.C., Langeheinecke, A., de Weerth, A., Schuchmann, M., Schaberg, T., Ligges, S., Eveslage, M., Hagen, R.M., Burchard, G.D., Lohse, A.W., Group, f.t.S.S., Ruf, B., Porschen, R., Trenn, G., Butterfaß-Bahloul, T., Wuerthwein, G., Oeder, F., Runge, A., Klauss, E., Hansen-Rosenblatt, N., Werner, T., Schulze, K., Kreuels, B., Schäfer, G., Hübener, P., Hennigs, A., Beisel, C., Fischer-Brügge, D., Zimmermann-Fraedrich, K., Röder, C., Grigo, N., Riecke, A., Schreckenbauer, H., Hemmer, C., Klammt, S., Geerdes-Fenge, H., Frimmel, S., Kittner, J.M., Rey, J.W., Schattenberg, J.M., Thieringer, F., Schmits, R., Grandt, D., Büch, P.M., Klebert, A., Mittag, M.A., Lehnen, S., Tiefengraber, D., Radecke, K., Hering, I., Zeller, W., Rundt, L., Brandt, L., Baltes, P., Dajani, D., Kurniawan, N., Pflüger, C., Behjat, N., Engel, U., Unger, M. and Group, f.t.S.S., 2016. Saccharomyces boulardii to prevent antibiotic-associated diarrhea: a randomized, double-masked, placebo-controlled trial. Open Forum Infectious Diseases 3: ofw011.
European Agency for the Evaluation of Medicinal Products (EMEA), 2001. Clavulanic acid. Report EMEA/MRL/776?01-Final. Committee for Veterinary Medicinal Products, London, pp. 1-6.

European Center for Disease Prevention and Control, 2018. Antimicrobial consumption - Annual Epidemiological Report for 2017. Annual Epidemiological Report on Ciommunicable Diseases in Europe. ECDC, Stockholm, Sweden.

Furet, J.P., Firmesse, O., Gourmelon, M., Bridonneau, C., Tap, J., Mondot, S., Dore, J. and Corthier, G., 2009. Comparative assessment of human and farm animal faecal microbiota using real-time quantitative PCR. FEMS Microbiology Ecology 68: 351-362.

Guarner, F., Sanders, M.E., Eliakim, R., Fedorak, R., Gangl, A., Garisch, J., Kaufmann, P., Karakan, T., Khan, A.G. and Kim, N., 2017. Probiotics and prebiotics. World Gastroenterology Organisation Global Guidelines. World Gastroenterology Organisation, Milwaukee, WI, USA. Available at: https://tinyurl.com/4sapeppc Guo, X., Xia, X., Tang, R., Zhou, J., Zhao, H. and Wang, K., 2008. Development of a real-time PCR method for Firmicutes and Bacteroidetes in faeces and its application to quantify intestinal population of obese and lean pigs. Letters in Applied Microbiology 47: 367-373.

Hartemink, R., Domenech, V.R. and Rombouts, F.M., 1997. LAMVAB - A new selective medium for the isolation of lactobacilli from faeces. Journal of Microbiological Methods 29: 77-84.

Hill, C., Guarner, F., Reid, G., Gibson, G.R., Merenstein, D.J., Pot, B., Morelli, L., Canani, R.B., Flint, H.J., Salminen, S., Calder, P.C. and Sanders, M.E., 2014. Expert consensus document. The International Scientific Association for Probiotics and Prebiotics consensus statement on the scope and appropriate use of the term probiotic. Nature Reviews Gastroenterology and Hepatology 11: 506-514.

Kalliomaki, M., Salminen, S., Arvilommi, H., Kero, P., Koskinen, P. and Isolauri, E., 2001. Probiotics in primary prevention of atopic disease: a randomised placebo-controlled trial. The Lancet 357: 1076-1079.

Klindworth, A., Pruesse, E., Schweer, T., Peplies, J., Quast, C., Horn, M. and Glockner, F.O., 2013. Evaluation of general 16S ribosomal RNA gene PCR primers for classical and next-generation sequencingbased diversity studies. Nucleic Acids Research 41: e1.

Kotowska, M., Albrecht, P. and Szajewska, H., 2005. Saccharomyces boulardii in the prevention of antibiotic-associated diarrhoea in children: a randomized double-blind placebo-controlled trial. Alimentary Pharmacology and Therapeutics 21: 583-590.

Kozich, J.J., Westcott, S.L., Baxter, N.T., Highlander, S.K. and Schloss, P.D., 2013. Development of a dual-index sequencing strategy and curation pipeline for analyzing amplicon sequence data on the MiSeq Illumina sequencing platform. Applied and Environmental Microbiology 79: 5112-5120.

Ladirat, S.E., Schoterman, M.H., Rahaoui, H., Mars, M., Schuren, F.H., Gruppen, H., Nauta, A. and Schols, H.A., 2014a. Exploring the effects of galacto-oligosaccharides on the gut microbiota of healthy adults receiving amoxicillin treatment. British Journal of Nutrition 112: 536-546.

Ladirat, S.E., Schuren, F.H., Schoterman, M.H., Nauta, A., Gruppen, H. and Schols, H.A., 2014b. Impact of galacto-oligosaccharides on the gut microbiota composition and metabolic activity upon antibiotic treatment during in vitro fermentation. FEMS Microbiology Ecology 87: 41-51. 
Lazo-Vélez, M.A., Serna-Saldívar, S.O., Rosales-Medina, M.F., Tinoco-Alvear, M. and Briones-García, M., 2018. Application of Saccharomyces cerevisiae var. boulardii in food processing: a review. Journal of Applied Microbiology 125: 943-951.

Legen, I., Kracun, M., Salobir, M. and Kerc, J., 2006. The evaluation of some pharmaceutically acceptable excipients as permeation enhancers for amoxicillin. International Journal of Pharmaceutics 308: 84-89.

Liu, L., Firrman, J., Tanes, C., Bittinger, K., Thomas-Gahring, A., Wu, G.D., Van den Abbeele, P. and Tomasula, P.M., 2018. Establishing a mucosal gut microbial community in vitro using an artificial simulator. PLoS ONE 13: e0197692.

Mantegazza, C., Molinari, P., D’Auria, E., Sonnino, M., Morelli, L. and Zuccotti, G.V., 2018. Probiotics and antibiotic-associated diarrhea in children: a review and new evidence on Lactobacillus rhamnosus GG during and after antibiotic treatment. Pharmacological Research 128: 63-72.

McFarland, L.V., 2008. Antibiotic-associated diarrhea: epidemiology, trends and treatment. Future Microbiology 3: 563-578.

McFarland, L.V. and Elmer, G.W., 2005. Properties of evidence-based probiotics for human health. In: Goktepe, I., Juneja, V.K. and Ahmedna, M. (eds.) Probiotics in food safety and human health. CRC Press, Boca Raton, FL, USA, pp. 110-128.

McFarland, L.V., Surawicz, C.M., Greenberg, R.N., Elmer, G.W., Moyer, K.A., Melcher, S.A., Bowen, K.E. and Cox, J.L., 1995. Prevention of beta-lactam-associated diarrhea by Saccharomyces boulardii compared with placebo. American Journal of Gastroenterology 90: 439-448.

Minekus, M., Alminger, M., Alvito, P., Ballance, S., Bohn, T., Bourlieu, C., Carrière, F., Boutrou, R., Corredig, M., Dupont, D., Dufour, C., Egger, L., Golding, M., Karakaya, S., Kirkhus, B., Le Feunteun, S., Lesmes, U., Macierzanka, A., Mackie, A., Marze, S., McClements, D.J., Ménard, O., Recio, I., Santos, C.N., Singh, R.P., Vegarud, G.E., Wickham, M.S., Weitschies, W. and Brodkorb, A., 2014. A standardised static in vitro digestion method suitable for food - an international consensus. Food and Function 5: 1113-1124.

Minekus, M., Smeets-Peeters, M., Bernalier, A., Marol-Bonnin, S., Havenaar, R., Marteau, P., Alric, M., Fonty, G. and Huis in't Veld, J.H., 1999. A computer-controlled system to simulate conditions of the large intestine with peristaltic mixing, water absorption and absorption of fermentation products. Applied Microbiology and Biotechnology 53: 108-114.

Moens, F., Duysburgh, C., van den Abbeele, P., Morera, M. and Marzorati, M., 2019. Lactobacillus rhamnosus GG and Saccharomyces cerevisiae boulardii exert synergistic antipathogenic activity in vitro against enterotoxigenic Escherichia coli. Beneficial Microbes 10: 923-935.

Molly, K., Vande Woestyne, M. and Verstraete, W., 1993. Development of a 5-step multi-chamber reactor as a simulation of the human intestinal microbial ecosystem. Applied Microbiology and Biotechnology 39: 254-258.

Moré, M.I. and Swidsinski, A., 2015. Saccharomyces boulardii CNCM I-745 supports regeneration of the intestinal microbiota after diarrheic dysbiosis - a review. Clinical and Experimental Gastroenterology 8: 237-255.
Neut, C., Mahieux, S. and Dubreuil, L.J., 2017. Antibiotic susceptibility of probiotic strains: Is it reasonable to combine probiotics with antibiotics? Médecine et Maladies Infectieuses 47: 477-483.

Palleja, A., Mikkelsen, K., Forslund, S., Kashani, A., Allin, K., Nielsen, T., Hansen, T., Feng, Q., Zhang, C., Pyl, P., Coelho, L.P., Yang, H., Wang, J., Typas, A., Frost, M., Nielsen, H., Bork, P., Wang, J. and Pedersen, O., 2018. Recovery of gut microbiota of healthy adults following antibiotic exposure. Nature Microbiology 3: 1255-1265.

Panda, S., El khader, I., Casellas, F., López Vivancos, J., García Cors, M., Santiago, A., Cuenca, S., Guarner, F. and Manichanh, C., 2014. Short-term effect of antibiotics on human gut microbiota. PLoS ONE 9: e95476.

Possemiers, S., Verthe, K., Uyttendaele, S. and Verstraete, W., 2004. PCR-DGGE-based quantification of stability of the microbial community in a simulator of the human intestinal microbial ecosystem. FEMS Microbiology Ecology 49: 495-507.

Pozzoni, P., Riva, A., Bellatorre, A.G., Amigoni, M., Redaelli, E., Ronchetti, A., Stefani, M., Tironi, R., Molteni, E.E., Conte, D., Casazza, G. and Colli, A., 2012. Saccharomyces boulardii for the prevention of antibiotic-associated diarrhea in adult hospitalized patients: a single-center, randomized, double-blind, placebocontrolled trial. American Journal of Gastroenterology 107: 922-931.

Ramamurthy, D., Pazhani, G.P., Sarkar, A., Nandy, R.K., Rajendran, K., Sur, D., Manna, B. and Ramamurthy, T., 2013. Case-control study on the role of enterotoxigenic Bacteroides fragilis as a cause of diarrhea among children in Kolkata, India. PLoS ONE 8: e60622.

Rinttila, T., Kassinen, A., Malinen, E., Krogius, L. and Palva, A., 2004. Development of an extensive set of $16 \mathrm{~S}$ rDNA-targeted primers for quantification of pathogenic and indigenous bacteria in faecal samples by real-time PCR. Journal of Applied Microbiology 97: 1166-1177.

Schloss, P.D. and Westcott, S.L., 2011. Assessing and improving methods used in operational taxonomic unit-based approaches for 16S rRNA gene sequence analysis. Applied and Environmental Microbiology 77: 3219-3226.

Soldi, S., Vasileiadis, S., Lohner, S., Uggeri, F., Puglisi, E., Molinari, P., Donner, E., Sieland, C., Decsi, T., Sailer, M. and Theis, S., 2019. Prebiotic supplementation over a cold season and during antibiotic treatment specifically modulates the gut microbiota composition of 3-6 year-old children. Beneficial Microbes 10: 253-263.

Sommer, F. and Bäckhed, F., 2013. The gut microbiota - masters of host development and physiology. Nature Reviews Microbiology 11: 227-238.

Spacova, I., O'Neill, C. and Lebeer, S., 2020. Lacticaseibacillus rhamnosus GG inhibits infection of human keratinocytes by Staphylococcus aureus through mechanisms involving cell surface molecules and $\mathrm{pH}$ reduction. Beneficial Microbes 11: 703-715.

Szajewska, H. and Kolodziej, M., 2015. Systematic review with meta-analysis: Lactobacillus rhamnosus GG in the prevention of antibiotic-associated diarrhoea in children and adults. Alimentary Pharmacology and Therapeutics 42: 1149-1157. 
Tremblay, A., Fatani, A., Ford, A.L., Piano, A., Nagulesapillai, V., Auger, J., MacPherson, C.W., Christman, M.C., Tompkins, T.A. and Dahl, W.J., 2020. Safety and effect of a low- and high-dose multi-strain probiotic supplement on microbiota in a general adult population: a randomized, double-blind, placebo-controlled study. Journal of Dietary Suppl. 18: 227-247.

Tsitko, I., Wiik-Miettinen, F., Mattila, O., Rosa-Sibakov, N., SeppänenLaakso, T., Maukonen, J., Nordlund, E. and Saarela, M., 2019. A small in vitro fermentation model for screening the gut microbiota effects of different fiber preparations. International Journal of Molecular Sciences 20: 1925.

Van de Wiele, T., Van den Abbeele, P., Ossieur, W., Possemiers, S. and Marzorati, M., 2015. The simulator of the human intestinal microbial ecosystem $\left(\right.$ SHIME $\left.^{\oplus}\right)$. In: Verhoeckx, K., Cotter, P., López-Expósito, I., Kleiveland, C., Lea, T., Mackie, A., Requena, T., Swiatecka D. and Wichers, H. (eds.), The impact of food bioactives on health: in vitro and ex vivo models. Springer International Publishing, Berlin, Germany, pp. 305-317.

Van den Abbeele, P., Belzer, C., Goossens, M., Kleerebezem, M., De Vos, W.M., Thas, O., De Weirdt, R., Kerckhof, F.M. and Van de Wiele, T., 2013. Butyrate-producing Clostridium cluster XIVa species specifically colonize mucins in an in vitro gut model. ISME Journal 7: 949-961.

Van den Abbeele, P., Grootaert, C., Marzorati, M., Possemiers, S., Verstraete, W., Gerard, P., Rabot, S., Bruneau, A., El Aidy, S., Derrien, M., Zoetendal, E., Kleerebezem, M., Smidt, H. and Van de Wiele, T., 2010. Microbial community development in a dynamic gut model is reproducible, colon region specific, and selective for Bacteroidetes and Clostridium cluster IX. Applied and Environmental Microbiology 76: 5237-5246.
Van den Abbeele, P., Roos, S., Eeckhaut, V., MacKenzie, D.A., Derde, M., Verstraete, W., Marzorati, M., Possemiers, S., Vanhoecke, B., Van Immerseel, F. and Van de Wiele, T., 2012. Incorporating a mucosal environment in a dynamic gut model results in a more representative colonization by lactobacilli. Microbial Biotechnology 5: 106-115.

Van den Abbeele, P., Taminiau, B., Pinheiro, I., Duysburgh, C., Jacobs, H., Pijls, L. and Marzorati, M., 2018. Arabinoxylo-oligosaccharides and inulin impact inter-individual variation on microbial metabolism and composition, which immunomodulates human cells. Journal of Agricultural and Food Chemistry 66: 1121-1130.

Van Herreweghen, F., Van den Abbeele, P., De Mulder, T., De Weirdt, R., Geirnaert, A., Hernandez-Sanabria, E., Vilchez-Vargas, R., Jauregui, R., Pieper, D.H., Belzer, C., De Vos, W.M. and Van de Wiele, T., 2017. In vitro colonisation of the distal colon by Akkermansia muciniphila is largely mucin and pH dependent. Beneficial Microbes 8: 81-96. Vanderhoof, J.A., Whitney, D.B., Antonson, D.L., Hanner, T.L., Lupo, J.V. and Young, R.J., 1999. Lactobacillus GG in the prevention of antibiotic-associated diarrhea in children. Journal of Pediatrics 135: 564-568.

Wang, Q., Garrity, G.M., Tiedje, J.M. and Cole, J.R., 2007. Naive Bayesian classifier for rapid assignment of rRNA sequences into the new bacterial taxonomy. Applied and Environmental Microbiology 73: 5261-5267.

White, A.R., Kaye, C., Poupard, J., Pypstra, R., Woodnutt, G. and Wynne, B., 2004. Augmentin ${ }^{\oplus}$ (amoxicillin/clavulanate) in the treatment of community-acquired respiratory tract infection: a review of the continuing development of an innovative antimicrobial agent. Journal of Antimicrobial Chemotherapy 53: i3-i20. 
